\title{
Effects of N and/or S Doping on Structure and Photocatalytic Properties of BiOBr Crystals
}

\author{
Guo-Hua Jiang $\cdot$ Xia Li $\cdot$ Zhen Wei $\cdot$ Teng-Teng Jiang $\cdot$ Xiang-Xiang Du $\cdot$ Wen-Xing Chen
}

Received: 16 April 2014/Revised: 5 September 2014/Published online: 22 January 2015

(C) The Chinese Society for Metals and Springer-Verlag Berlin Heidelberg 2015

\begin{abstract}
The $\mathrm{BiOBr}$ crystals doped with $\mathrm{N}$ and/or $\mathrm{S}$ have been prepared by a facile and rapid solvothermal method using urea and/or thiourea as doped resource. The morphologies and structures of the as-prepared samples were characterized by SEM, TEM, XRD, XPS and PL spectrum. Their electronic structures have been investigated and discussed using first principles based on the density functional theory. The photocatalytic properties of the products can be adjusted by the content and species of dopants.
\end{abstract}

KEY WORDS: Hydrothermal method; Photocatalysis; Microstructure and properties

\section{Introduction}

Bismuth oxyhalides [BiOX $(X=\mathrm{Cl}, \mathrm{Br}, \mathrm{I})]$, crystallizing in the tetragonal matlockite structure, have been investigated as potential candidates for photocatalytic applications due to their high photocatalytic activity and stability under UV and visible light irradiation [1-3]. $\mathrm{BiOBr}$, a semiconductor material with the unique sandwich-like tetragonal matlockite structure, a layered structure composed of $\left[\mathrm{Bi}_{2} \mathrm{O}_{2}\right]^{2+}$ layers interleaved with double $\mathrm{Br}$ layers to form a $\left[\mathrm{Bi}_{2} \mathrm{O}_{2} \mathrm{Br}_{2}\right]$ layer unit, has been attracted considerable attention for its excellent performances as visible lightinduced photocatalyst $[4,5]$.

Available online at http://link.springer.com/journal/40195

G.-H. Jiang $(\bowtie) \cdot$ X. Li $\cdot$ Z. Wei · T.-T. Jiang $\cdot$ X.-X. Du

W.-X. Chen

Key Laboratory of Advanced Textile Materials and Manufacturing Technology (ATMT), Ministry of Education,

Zhejiang Sci-Tech University, Hangzhou 310018, China

e-mail: ghjiang_cn@zstu.edu.cn

G.-H. Jiang · W.-X. Chen

Department of Materials Engineering, College of Materials and

Textile, Zhejiang Sci-Tech University, Hangzhou 310018, China
Morphology and structure are the important and hot issues in photocatalysis. Small size and large surface area normally correspond to high photocatalytic activity. Lowdimensional nanostructures with high aspect ratio and porous nanostructures favorably affect charge and mass transport. Energy band engineering is also a fundamental aspect for the design and fabrication of semiconductor photocatalysts because the energy band configuration of a semiconductor plays a significant role in the determining of light absorption and redox potentials [6-8]. The most popular approach for tailoring the absorption edges of photocatalysts is to dope the host material with foreign species [9-12]. The metal-doped $\mathrm{BiOBr}$ photocatalysts exhibit outstanding activities under visible light irradiation. Liu et al. [13] reported that Al doping could greatly improve photocatalytic performance of $\mathrm{BiOBr}$. It was also confirmed in our previous experimental results that Tidoped $\mathrm{BiOBr}$ [14], Fe-doped $\mathrm{BiOBr}$ [15] and Mn-doped $\mathrm{BiOBr}$ [16] exhibited outstanding activities under visible light irradiation. They all showed that the optical response of doped $\mathrm{BiOBr}$ could have a red shift and the band gap of the doped $\mathrm{BiOBr}$ could be narrow. It has been recognized that codoping could greatly improve the photocatalytic activity which could be ascribed to the synergistic effects of the dopants. Likewise, the codoping of nonmetal 


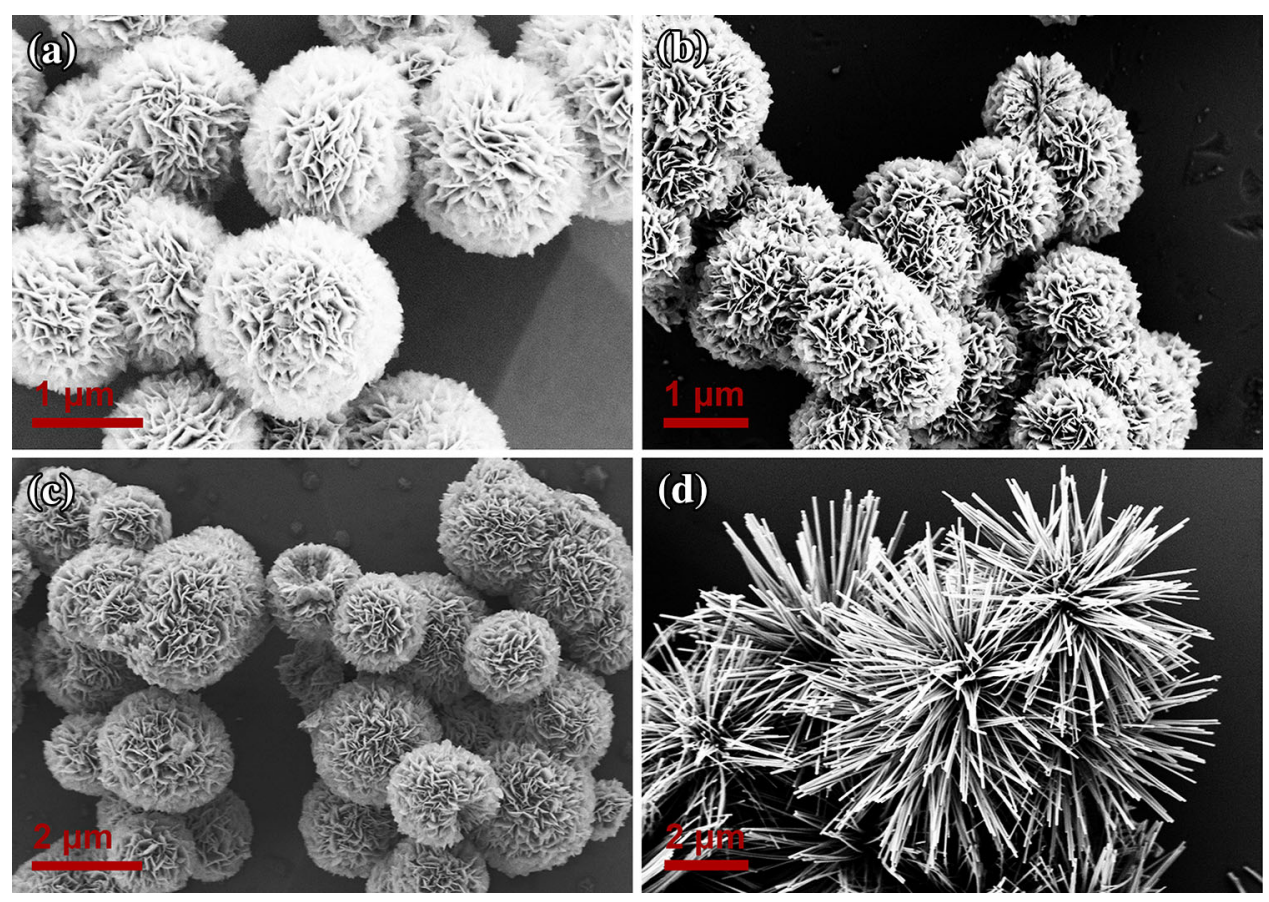

Fig. 1 SEM images of doped $\mathrm{BiOBr}$ samples prepared using different urea/thiourea mixtures: a $0 \mathrm{~g} \mathrm{urea} / 0 \mathrm{~g}$ thiourea, b $0.2 \mathrm{~g}$ urea/ $0 \mathrm{~g}$ thiourea, c $0.2 \mathrm{~g}$ urea/ $0.05 \mathrm{~g}$ thiourea, $\mathbf{d} 0 \mathrm{~g}$ urea/ $/ 0.5 \mathrm{~g}$ thiourea
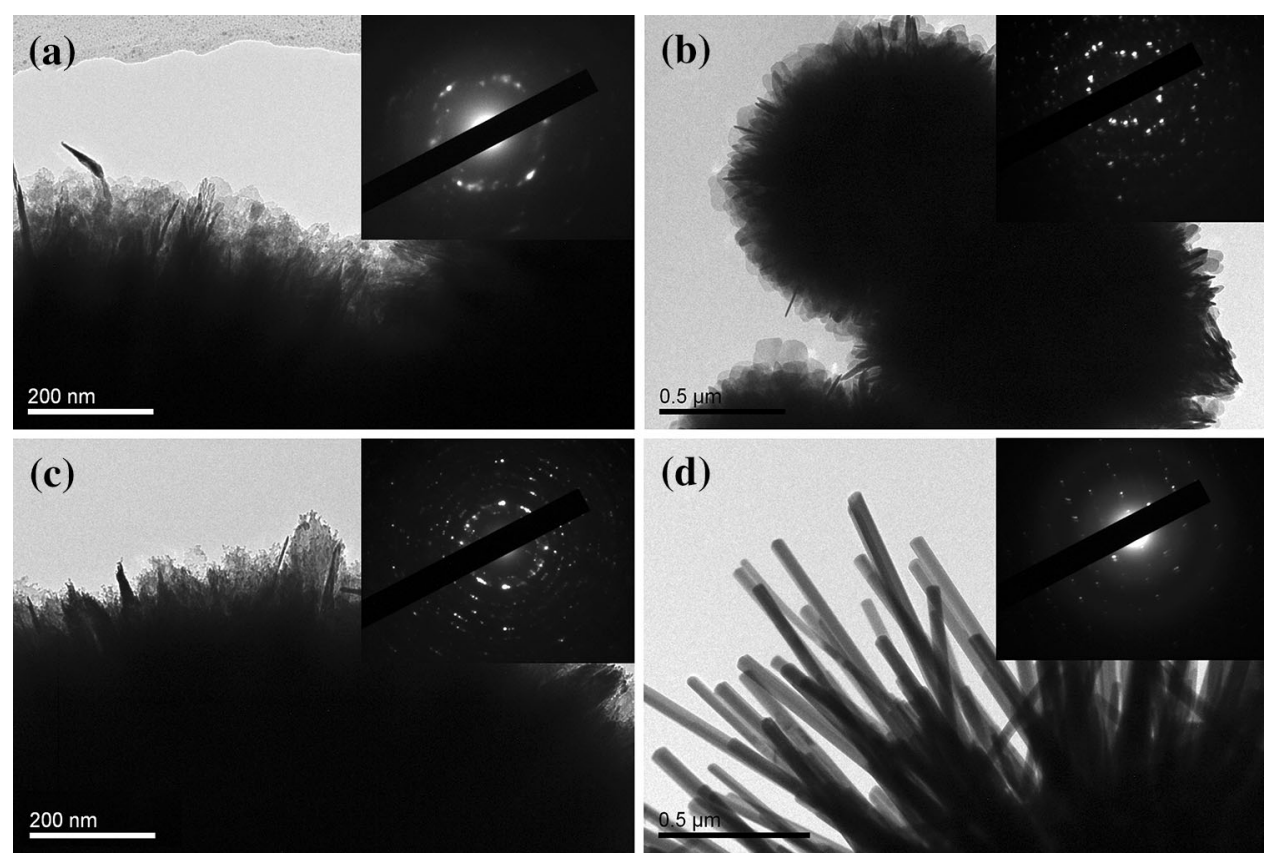

Fig. 2 TEM images of doped $\mathrm{BiOBr}$ samples prepared using different urea/thiourea mixtures: a $0 \mathrm{~g} \mathrm{urea} / 0 \mathrm{~g}$ thiourea; $\mathbf{b} 0.2 \mathrm{~g}$ urea/ $0 \mathrm{~g}$ thiourea; c $0.2 \mathrm{~g}$ urea/ $0.05 \mathrm{~g}$ thiourea; $\mathbf{d} 0 \mathrm{~g}$ urea/ $/ 0.5 \mathrm{~g}$ thiourea

dopants (N, S and C) into BiOBr nanocrystals also can reduce the recombination centers because codoping can passivate the defect bands produced by monodoping [17, 18]. Herein, visible light-induced $\mathrm{BiOBr}$ photocatalysts doped with $\mathrm{N}$ and/or $\mathrm{S}$ elements have been prepared by a facile and rapid solvothermal method. The physicochemical properties of doped $\mathrm{BiOBr}$ photocatalysts have been investigated by experimental and theoretical analysis. 


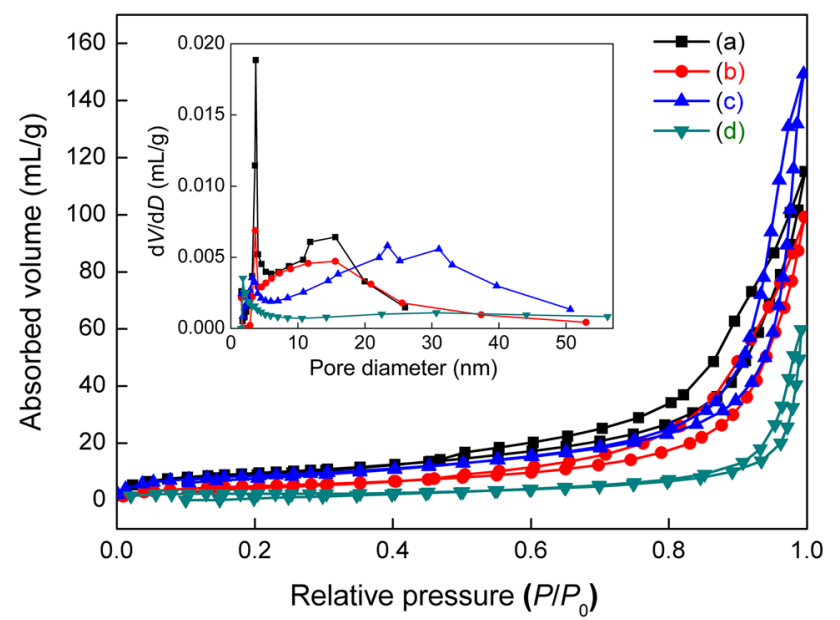

Fig. $3 \mathrm{~N}_{2}$ adsorption-desorption isotherms and corresponding pore size distribution curves of doped $\mathrm{BiOBr}$ samples prepared using different urea/thiourea mixtures: $a 0 \mathrm{~g}$ urea/ $0 \mathrm{~g}$ thiourea; $b 0.2 \mathrm{~g}$ urea/ $0 \mathrm{~g}$ thiourea; $c 0.2 \mathrm{~g}$ urea/ $0.05 \mathrm{~g}$ thiourea; $d 0 \mathrm{~g}$ urea/ $/ 0.5 \mathrm{~g}$ thiourea

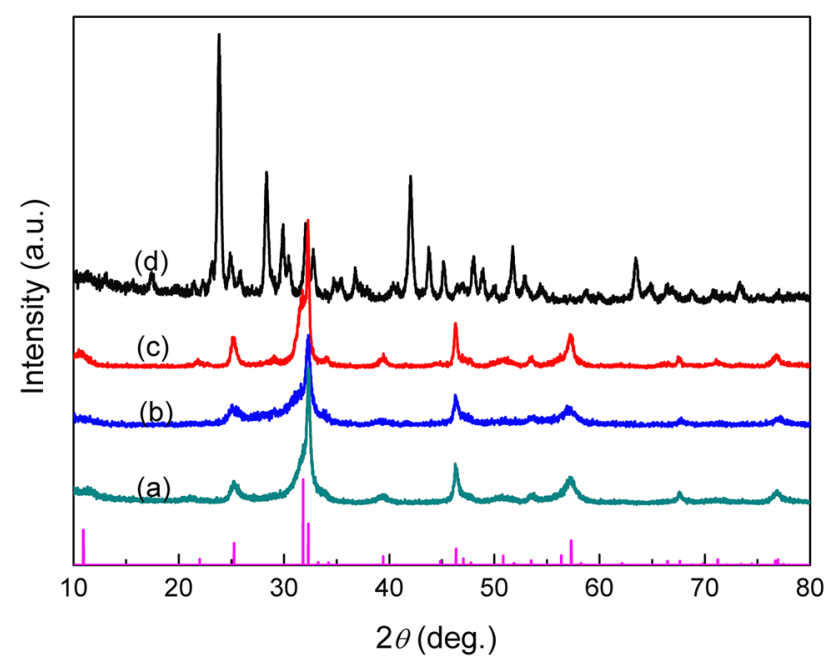

Fig. 4 XRD patterns of doped $\mathrm{BiOBr}$ samples prepared using different urea/thiourea mixtures: $a 0 \mathrm{~g}$ urea/ $/ \mathrm{g}$ thiourea; $b 0.2 \mathrm{~g}$ urea/ $0 \mathrm{~g}$ thiourea; $c 0.2 \mathrm{~g}$ urea/ $/ 0.05 \mathrm{~g}$ thiourea; $d 0 \mathrm{~g}$ urea/ $/ .5 \mathrm{~g}$ thiourea

\section{Experimental}

\subsection{Preparation of Materials}

Appropriate amounts of $\mathrm{Bi}\left(\mathrm{NO}_{3}\right)_{3} \cdot 5 \mathrm{H}_{2} \mathrm{O}(1 \mathrm{~g})$ were added to $60 \mathrm{~mL} \mathrm{2-methoxyethanol.} \mathrm{Then,} \mathrm{different} \mathrm{amounts} \mathrm{of}$ urea/thiourea (A: 0 g/0 g, B: 0.2 g/0 g, C: 0.2 g/0.05 g, D: $0 \mathrm{~g} / 0.5 \mathrm{~g}$ ) and $0.60 \mathrm{~g}$ CTAB were added with sonication to form a homogeneous solution. Then, the resultant solution was transferred into a 100-mL autoclave Teflon vessel, and the precursor suspension was then solvothermal treated at $180{ }^{\circ} \mathrm{C}$ for $48 \mathrm{~h}$. After cooling to room temperature, the precipitates were filtered and washed with deionized water and ethanol six times. The products were dried in a vacuum oven at $60{ }^{\circ} \mathrm{C}$ for $12 \mathrm{~h}$ to get the fine photocatalysts.

\subsection{Characterization}

The structures and crystal phase of the as-prepared samples were analyzed with a SIEMENS Diffraktometer D5000 $\mathrm{X}$-ray diffractometer with $\mathrm{Cu} K_{\alpha}$ radiation source at $35 \mathrm{kV}$, with a scan rate of 4 degree/s in the $2 \theta$ range of $10^{\circ}-80^{\circ}$. X-ray photoelectron spectroscopy (XPS) data were obtained with an obtained with an ESCALab220i-XL electron spectrometer from VG Scientific using $300 \mathrm{~W}$ $\mathrm{Al} K_{\alpha}$ radiation. The base pressure was about $3 \times 10^{-7} \mathrm{~Pa}$. The binding energies were referenced to the $\mathrm{C} 1 \mathrm{~s}$ line at $284.6 \mathrm{eV}$ from adventitious carbon. The ULTRA-55 fieldemission scanning electron microscope (FE-SEM) equipped with an energy-dispersive X-ray spectrum (EDS, Inca Energy-200) at an accelerating voltage of $10 \mathrm{kV}$. JSM2100 transmission electron microscope (TEM) was used to characterize the morphology of the as-prepared samples. The Brunauer-Emmet-Teller (BET) specific surface area was measured using a specific surface and pore size analysis instrument (3H-2000PS1/2 static volume method, China). The photoluminescence (PL) spectra were texted by HITACHI F-4600 fluorescence spectrophotometer. The band structures and densities of states have been calculated by Vienna Ab-initio Simulation Package (VASP) based on the density functional theory (DFT).

\subsection{Measurement of Photocatalytic Activity}

The photocatalytic activity of the samples was evaluated by the photodegradation of $\mathrm{RhB}$ in aqueous solution under visible light irradiation with an 11-W daylight lamp ( $25 \mathrm{~Hz}$ ) equipped with UV cutoff filter to provide visible light $(\lambda \geq 400 \mathrm{~nm}$ ). Before irradiation, $20 \mathrm{mg}$ as-prepared catalysts were added into $50 \mathrm{~mL} \mathrm{RhB}$ solution under magnetic stirring for $1 \mathrm{~h}$ to ensure the adsorption-desorption equilibrium. Then, the solution was stirred and exposed to the visible light irradiation. An amount of aliquots were taken away and separated as-prepared catalysts with centrifugation at the designated time. The upper layer was appropriately diluted, and the $\mathrm{RhB}$ concentrations (C) were measured by JASCO V-570 UV-Vis-NIR spectrophotometer (Japan).

\section{Results and Discussion}

Figure 1 shows the SEM images of doped $\mathrm{BiOBr}$ samples prepared using different urea/thiourea mixtures as doped resource. As shown in Fig. 1a, the flower-like pure BiOBr 

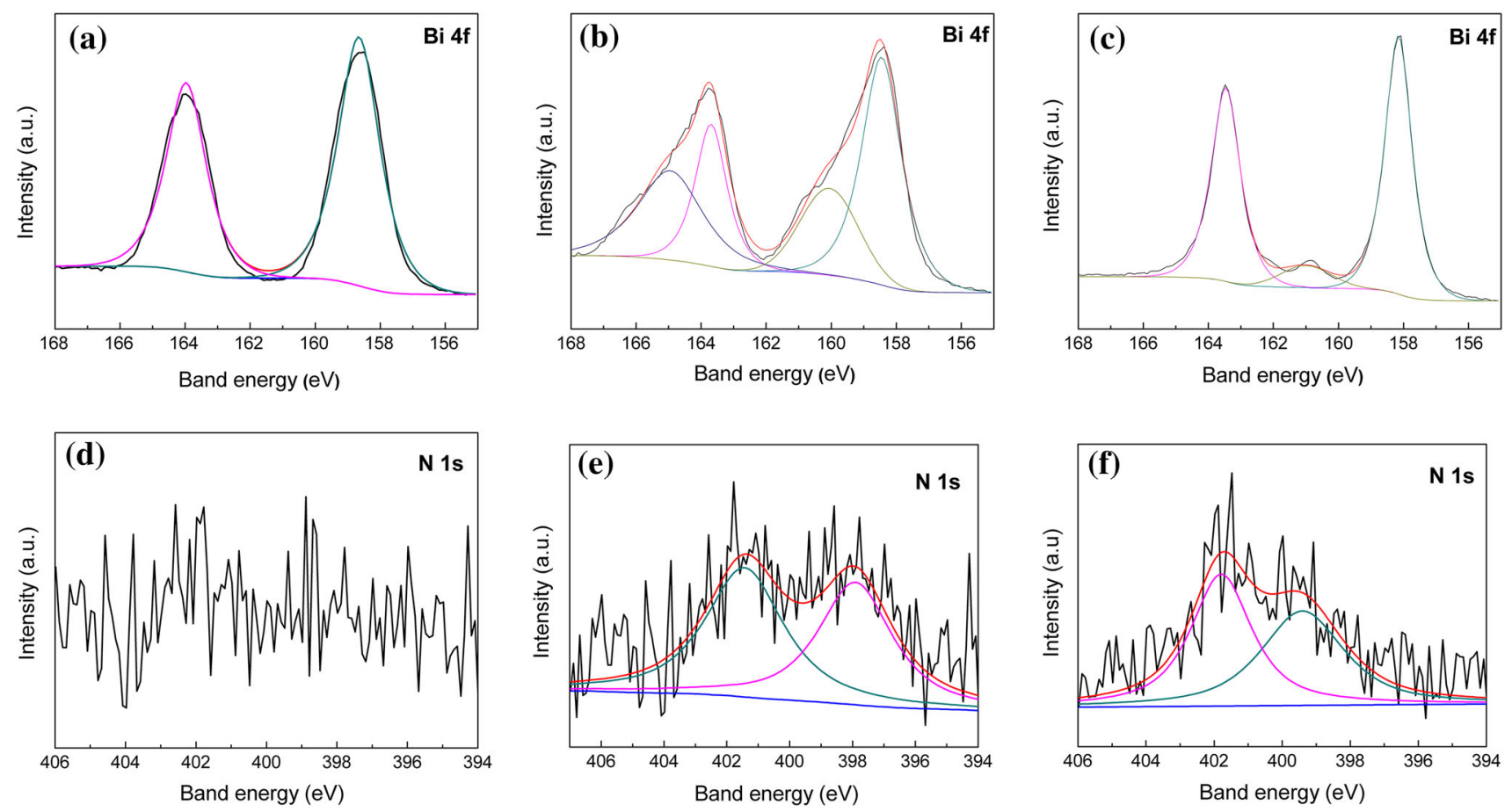

Fig. 5 The high-resolution XPS spectra of $\mathrm{Bi} 4 \mathrm{f}(\mathrm{S} 2 \mathrm{p})$ and $\mathrm{N} 1 \mathrm{~s}$ of $\mathrm{BiOBr}$ samples prepared using different urea/thiourea mixtures: a, d $0 \mathrm{~g}$ urea/0 $\mathrm{g}$ thiourea; $\mathbf{b}, \mathbf{e} 0.2 \mathrm{~g}$ urea/0.05 $\mathrm{g}$ thiourea; $\mathbf{c}, \mathbf{f} 0 \mathrm{~g}$ urea/ $/ 0.5 \mathrm{~g}$ thiourea

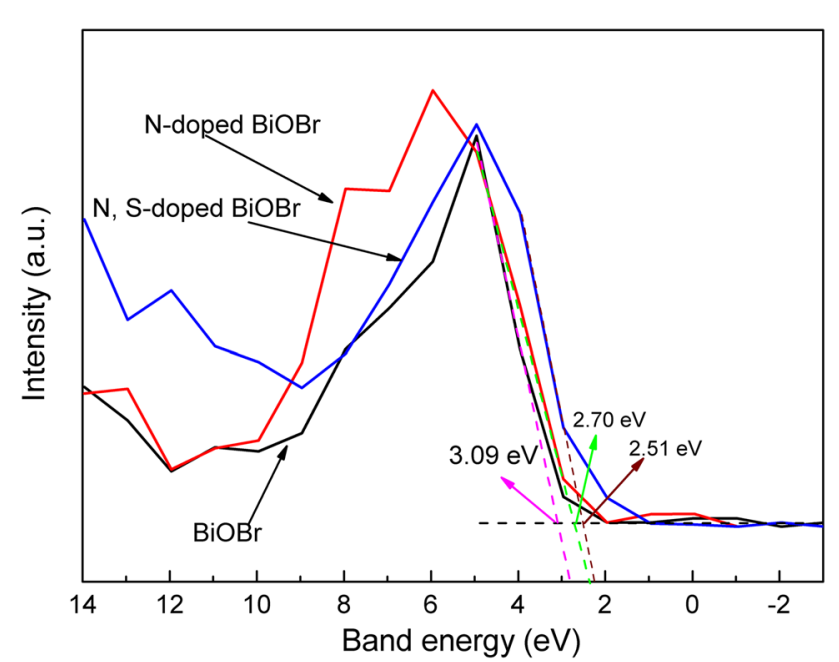

Fig. 6 XPS valence spectra showing the valence bands $V B$ of both pure $\mathrm{BiOBr}$ and $\mathrm{N}$ and/or S-doped $\mathrm{BiOBr}$

microspheres with diameter of $\sim 1.5 \mu \mathrm{m}$ can be observed in the absence of urea and thiourea. Only using urea as doped resource, the morphology of the products has no great change with pure BiOBr (Fig. 1b). Adding small amount of thiourea as codoped resource, the size uniformity of the resultant microspheres decreased (range from 0.5 to $2.5 \mu \mathrm{m}$ ) although the molecular structure of urea is close to that of thiourea (Fig. 1c). Interesting, the rod-like products are obtained only using thiourea as doped resource (Fig. 1d). It implies that the doped resource plays an important role for the formation of microsphere structure. Undoped and doped microspheres are assembled by many nanosheets. Selected area electron diffraction (SAED) patterns taken from the edge of the nanosheets reveal that these microspheres have a poly-crystalline nature. However, the nanorods obtained using thiourea as doped resource has a single-crystalline nature, as shown in Fig. 2. Figure 3 shows the nitrogen adsorption-desorption isotherms and the corresponding pore size distribution curves, which were carried to further investigate the porous structure of the products. The doped BiOBr microspheres have the higher specific surface area and pore volume compared to that of pure $\mathrm{BiOBr}$, owing to the pore been produced by the association of the smaller nanosheets which has a positive role for improvement of photocatalytic activity.

Figure 4 shows the XRD patterns of doped $\mathrm{BiOBr}$ samples prepared using different urea/thiourea mixtures as doped resource. All the diffraction peaks of products that prepared in the absence of urea and thiourea can be well matched with the tetragonal phase of $\mathrm{BiOBr}$ (JCPDS no. 73-2061) (Fig. 4a). Using urea as doped resource, the diffraction peaks of products still well matched with the tetragonal phase of $\mathrm{BiOBr}$ (Fig. 4b). Adding small amount of thiourea as codoped resource, the weak impurity peaks at $2 \theta=30^{\circ}, 38^{\circ}$ and $54^{\circ}$ can be founded due to the low content and high dispersity of 
dopants (Fig. 4c) [19]. Compared with the above samples, new diffraction peaks in the product that prepared only using thiourea as doped resource can be observed due to the formation of large amount of $\mathrm{Bi}_{2} \mathrm{~S}_{3}$ crystals which is completely different from $\mathrm{BiOBr}$ (Fig. 4d) [20, 21].

Figure 5 shows the high-resolution XPS spectra of Bi $4 \mathrm{f}$ ( $\mathrm{S} 2 \mathrm{p}$ ) and $\mathrm{N} 1 \mathrm{~s}$ of $\mathrm{BiOBr}$ samples. It can be seen from
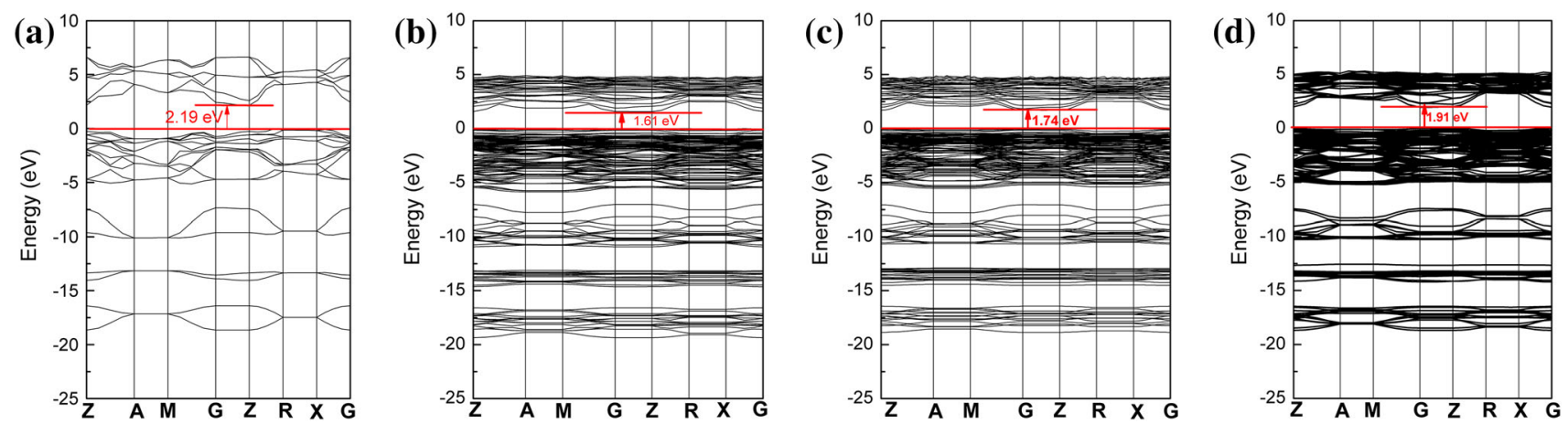

Fig. 7 Band structures of doped $\mathrm{BiOBr}$ samples prepared using different urea/thiourea mixtures: $\mathbf{a} 0 \mathrm{~g}$ urea/ $0 \mathrm{~g}$ thiourea; $\mathbf{b} 0.2 \mathrm{~g}$ urea/ $/ 0 \mathrm{~g}$ thiourea; c $0.2 \mathrm{~g}$ urea/0.05 g thiourea; $\mathbf{d} 0 \mathrm{~g}$ urea/0.5 $\mathrm{g}$ thiourea
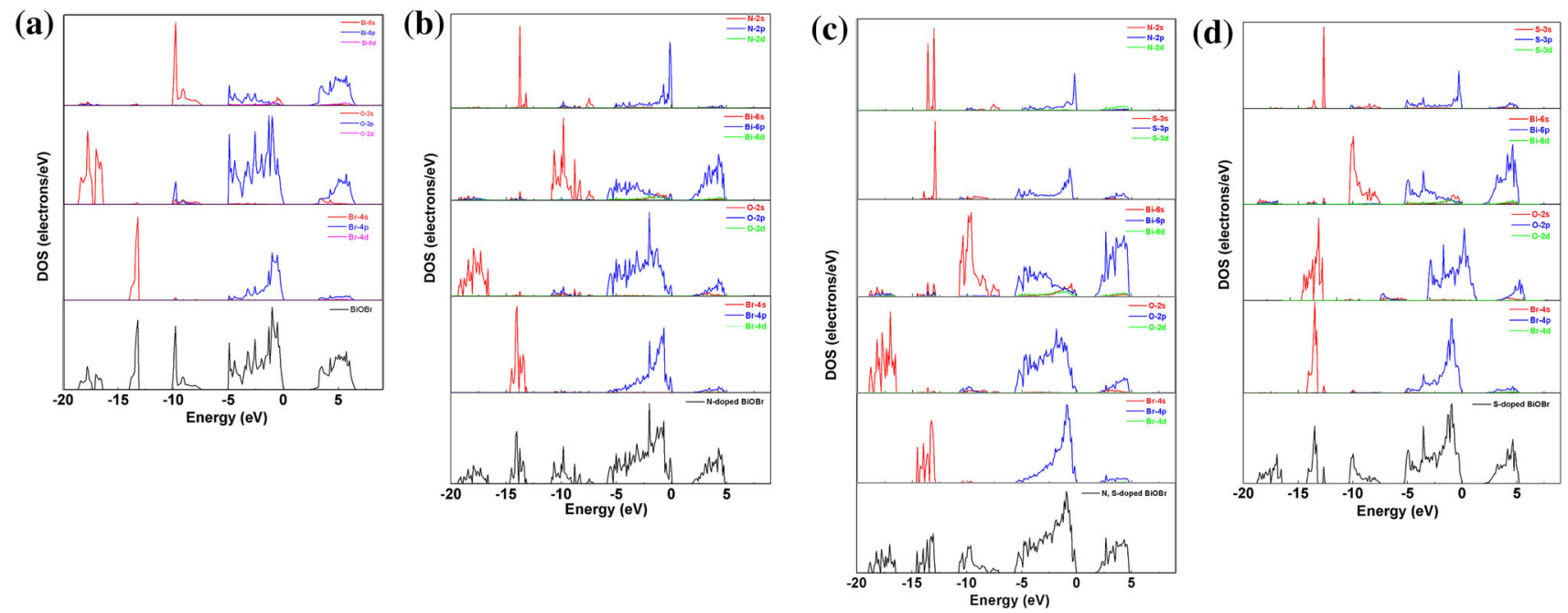

Fig. 8 Density of states $D O S$ and partial density of states $P D O S$ for of doped BiOBr samples prepared using different urea/thiourea mixtures: a $0 \mathrm{~g}$ urea/ $0 \mathrm{~g}$ thiourea; $\mathbf{b} 0.2 \mathrm{~g}$ urea/ $/ 0 \mathrm{~g}$ thiourea; $\mathbf{c} 0.2 \mathrm{~g}$ urea/ $0.05 \mathrm{~g}$ thiourea; $\mathbf{d} 0 \mathrm{~g}$ urea/ $/ .5 \mathrm{~g}$ thiourea

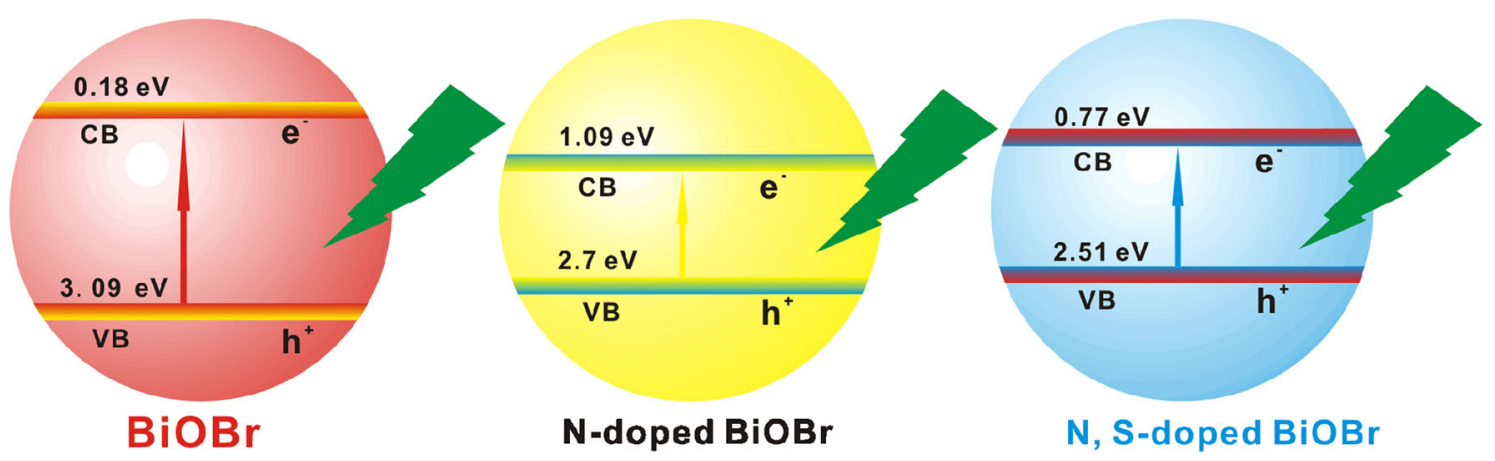

Fig. 9 Schematic illustration of both pure $\mathrm{BiOBr}$ and $\mathrm{N}$ - and/or S-doped $\mathrm{BiOBr}$ are measured by XPS valence spectra 
Fig. 5a, the high-resolution XPS spectrum of $\mathrm{Bi} 4 \mathrm{f}$ can be fitted as two peaks at binding energies of 158.6 and $163.9 \mathrm{eV}$ which are assigned to $\mathrm{Bi} 4 \mathrm{f}_{7 / 2}$ and $\mathrm{Bi} 4 \mathrm{f}_{5 / 2}$, respectively [22]. After only adding urea as doped resource, a new fitted peak appear at higher binding energy $(\sim 165.1$ and $159.9 \mathrm{eV})$. It can be ascribed to a slight surface charging effect for the polarization change of the crystals (Fig. 5b) [23]. In the case of products prepared in the presence of thiourea, the appearance of a new fitted peak at $\sim 161.0 \mathrm{eV}$ (S 2p) might be associated with strong chemical bonding and the effect of S doping (Fig. 5c). No signal of $\mathrm{N} 1 \mathrm{~s}$ in pure $\mathrm{BiOBr}$ crystals can be observed (Fig. 5d). However, two peaks at binding energies of 397.8 and $401.7 \mathrm{eV}$ are observed (Fig. 5e), indicating the presence of $\mathrm{N}$ dopant using urea or thiourea as doped resource [24]. Only using thiourea as doped resource, the relative peak intensity at $401.7 \mathrm{eV}$ is higher than that at $397.8 \mathrm{eV}$

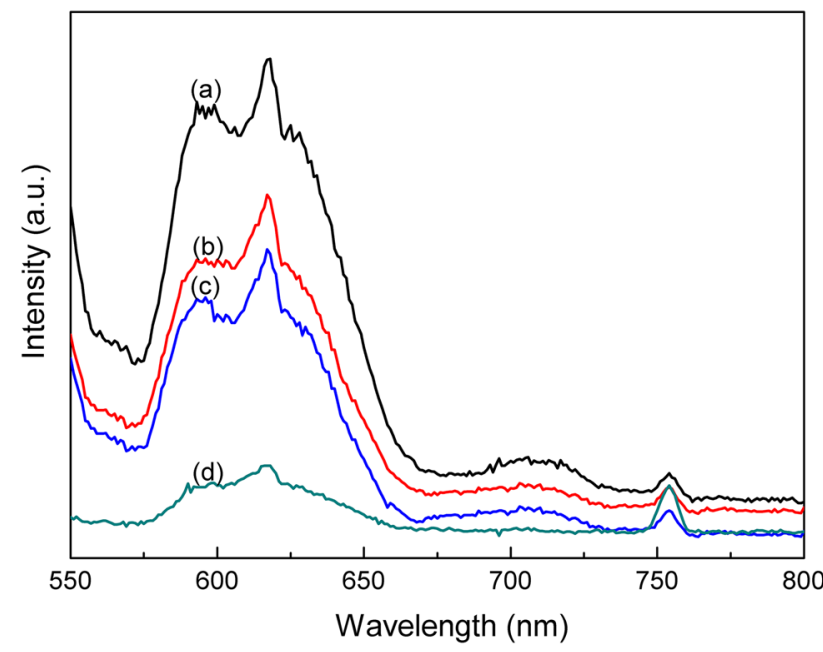

Fig. 10 Photoluminescence $P L$ emission spectra of doped $\mathrm{BiOBr}$ samples prepared using different urea/thiourea mixtures: a $0 \mathrm{~g}$ urea/ $0 \mathrm{~g}$ thiourea; b $0.2 \mathrm{~g}$ urea/ $0 \mathrm{~g}$ thiourea; $\mathbf{c} 0.2 \mathrm{~g}$ urea/ $/ 0.05 \mathrm{~g}$ thiourea; d $0 \mathrm{~g}$ urea/ $0.5 \mathrm{~g}$ thiourea

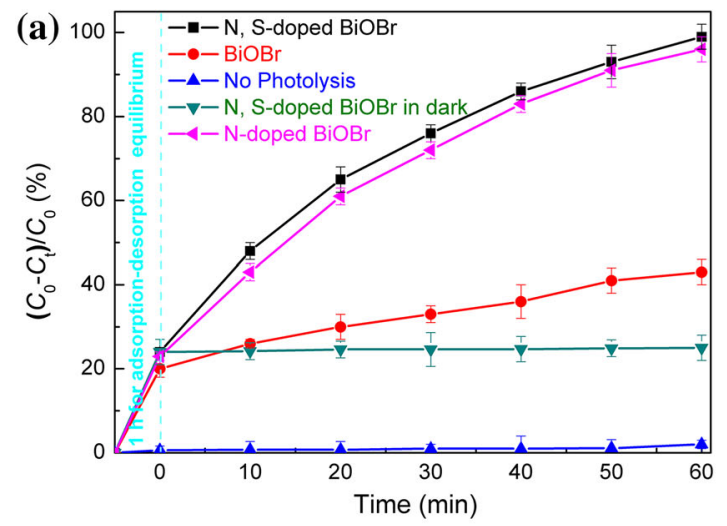

(Fig. 5f). It implies the some $\mathrm{N}$ atoms in the $\mathrm{BiOBr}$ crystal have been taken place by $\mathrm{S}$ atoms to form $\mathrm{N}$ - and S-codoped structure. Furthermore, the valence bands (VBs) of both pure $\mathrm{BiOBr}$ and $\mathrm{N}-$ - S-doped $\mathrm{BiOBr}$ are measured by XPS valence spectra [25]. From the valence bands (VBs) of both pure $\mathrm{BiOBr}$ and $\mathrm{N}$ - and/or S-doped $\mathrm{BiOBr}$ which are measured by XPS valence spectra (Fig. 6), it can be found that the $\mathrm{N}$-, S-doped $\mathrm{BiOBr}$ display the lowest valence band (VB) with the edge of the maximum energy at about $2.51 \mathrm{eV}$.

To investigate the electronic properties of the as-prepared products, the band structures with the same energy range of $-25-10 \mathrm{eV}$ have been calculated; as shown in Fig. 7, the Fermi energy $E_{\mathrm{F}}$ is set at $0 \mathrm{eV}$. It can be found that the valence band maximum (VBM) and the conduction band minimum (CBM) for the four crystals located at the different $\mathrm{k}$-space point positions, indicating that the four crystals have the different indirect transition band gap characteristic. The calculated minimum indirect band gaps for four crystals are 2.19, 1.61, 1.74 and $1.91 \mathrm{eV}$, respectively. The $\mathrm{N}$ and/or $\mathrm{S}$-doped $\mathrm{BiOBr}$ products exhibit lower band gaps than that of pure BiOBr. It is well known that the indirect band gap semiconductor helps to obtain excellent photocatalytic activity because the excited electrons must be emitted to conduction band by traveling certain k-space distance, which reduces the recombination probability of the photogenerated electrons and holes [26-28]. The total density of states and partial density of states of the products have been illustrated according with the energy range of band structure, as shown in Fig. 8. Compared the distribution of $\mathrm{VB}$ of $\mathrm{BiOBr}$, the $\mathrm{N}$ and/or $\mathrm{S}$ doping has the significant effect on the excited state density of $\mathrm{Bi}$ and $\mathrm{Br}$. Combined the results of calculated minimum indirect band gap and the valence band test, the minimum $\mathrm{CB}$ of doped $\mathrm{BiOBr}$ up-shifts by $-0.91 \mathrm{eV}$ and occurs at 1.09 or $-0.59 \mathrm{eV}$ and occurs at $0.77 \mathrm{eV}$ compared to that of pure $\mathrm{BiOBr}$ at $0.18 \mathrm{eV}$ (Fig. 9). This indicates that mobility of

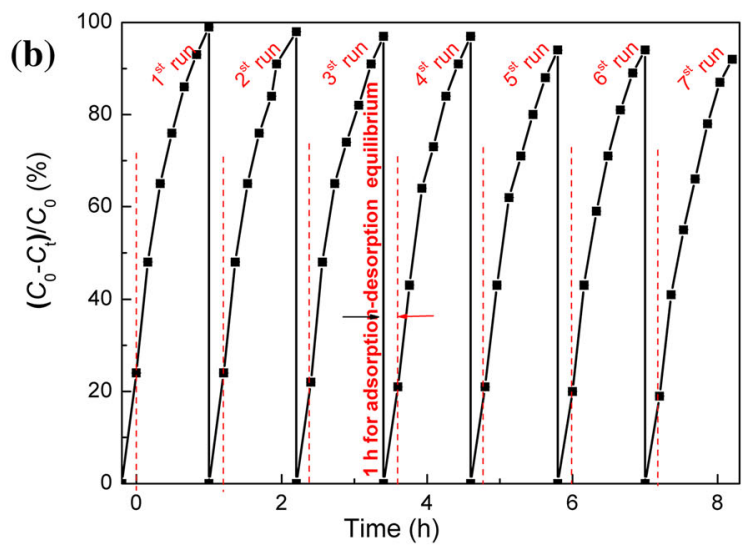

Fig. 11 Photocatalytic degradation of RhB over different photocatalytic materials a cycling runs for the photodegradation of RhB over asprepared N-, S-codoped $\mathrm{BiOBr} \mathbf{b}$ 
photoexcited electrons is low, suggesting that structure effects of distorted tetragonal $\mathrm{BiOBr}$ play an important role in photocatalysis [29]. Figure 10 shows the photoluminescence (PL) emission spectra of doped BiOBr samples, a broad PL emission spectrum was observed for all products. The spectrum indicated that most of the charges quickly recombine in $\mathrm{BiOBr}$ to produce $\mathrm{PL}$ emission. In contrast, the $\mathrm{PL}$ intensity of doped $\mathrm{BiOBr}$ reduces evidently. It implies the electron-hole recombination on the surface of catalysts is largely inhibited to generate more photoelectrons and holes to participate in the photocatalytic reactions [22].

The photocatalytic activity of the as-prepared products was further evaluated by degradation of Rhodamine B $(\mathrm{RhB})$ under the visible light irradiation, as shown in Fig. 11a. The adsorption-desorption equilibrium for photocatalysts can be reached after stirring for $1 \mathrm{~h}$ in the dark. Neither photolysis (without photocatalyst) nor catalysis (without light) exhibited degradation ability on $\mathrm{RhB}$, suggesting that degradation ability is induced by photocatalysis. The RhB could be completely degraded by $\mathrm{N}$-, $\mathrm{S}$-doped $\mathrm{BiOBr}$ photocatalysts within $1 \mathrm{~h}$ and $\mathrm{N}$-doped $\mathrm{BiOBr}$ photocatalysts with a longer time. However, only about $45 \%$ of $\mathrm{RhB}$ is degraded over $\mathrm{BiOBr}$ after irradiation for $1 \mathrm{~h}$. The relatively higher photocatalytic activity of doped $\mathrm{BiOBr}$ photocatalysts may result from the doped of foreign species, which is beneficial to improve of photoadsorption property, reduce band gap and enlarge specific surface area and the pore volume [30, 31]. In addition, the recycling experiments were performed to evaluate the durability of the $\mathrm{N}-$, S-doped $\mathrm{BiOBr}$ photocatalysts. As shown in Fig. 11b, RhB can be completely degraded after 7 runs ( $1 \mathrm{~h}$ for each run), indicating the good durability of $\mathrm{N}-$, S-doped $\mathrm{BiOBr}$ photocatalysts.

\section{Conclusions}

The BiOBr crystals doped with $\mathrm{N}$ and/or $\mathrm{S}$ elements have been prepared by a facile and rapid solvothermal method using urea and/or thiourea as doped resource. The morphology, structural, electronic and optical properties of the products have been investigated by experimental and theoretical analysis. The size and morphologies of the products can be adjusted using different doped resources. The structural, electronic and optical properties of the samples also can be affected by the content and species of dopants. The $\mathrm{N}$-, S-codoped BiOBr products showed the highest photocatalytic activity and good durability.

Acknowledgments This work was financially supported by the "521 Talents Training Plan" in ZSTU, the National Natural Science Foundation of China (Nos. 51133006, 51373155), the Innovative Program for Graduate Students from ZSTU (No. YCX12013), the Training Programs for Excellent Master's Thesis from ZSTU (Nos.
2013YSPY14, 2012G0301009) and the National Training Programs of Innovation and Entrepreneurship for Undergraduates (No. 201310338004).

\section{References}

[1] K. Zhang, J. Liang, S. Wang, J. Liu, K. Ren, X. Zheng, H. Luo, Y. Peng, X. Zou, X. Bo, J. Li, X. Yu, Cryst. Growth Des. 12, 793 (2012)

[2] X. Zhang, Z. Ai, F. Jia, L. Zhang, J. Phys. Chem. C 112, 747 (2008)

[3] X. Zhang, L. Zhang, J. Phys. Chem. C 114, 18198 (2010)

[4] Z. Deng, D. Chen, B. Peng, F. Tang, Cryst. Growth Des. 8, 2995 (2008)

[5] X. Li, G. Jiang, Z. Wei, X. Wang, W. Chen, L. Shen, MRS Commun. 3, 219 (2013)

[6] H. Fan, H. Li, B. Liu, Y. Lu, T. Xie, D. Wang, ACS Appl. Mater. Interf. 4, 4853 (2012)

[7] X. Wang, Y. Tang, Z. Chen, T. Lim, J. Mater. Chem. 22, 23149 (2012)

[8] K. Maeda, K. Domen, J. Phys. Chem. Lett. 1, 2655 (2010)

[9] A. Ghicov, B. Schmidt, J. Kunze, P. Schmuki, Chem. Phys. Lett. 433, 323 (2007)

[10] E. Wang, T. He, L. Zhao, Y. Chen, Y. Cao, J. Mater. Chem. 21, 144 (2011)

[11] Y. Su, L. Hou, C. Du, L. Peng, K. Guan, X. Wang, RSC Adv. 2, 6266 (2012)

[12] F. Dong, Y. Sun, M. Fu, W. Ho, S. Lee, Z. Wu, Langmuir 28, 766 (2012)

[13] Z. Liu, B. Wu, Y. Zhao, J. Niu, Y. Zhu, Ceram. Int. 40, 5597 (2014)

[14] R. Wang, G. Jiang, X. Wang, R. Hu, X. Xi, Y. Zhou, S. Bao, T. Tong, Powder Technol. 228, 258 (2012)

[15] G. Jiang, X. Wang, Z. Wei, X. Li, X. Xi, R. Hu, B. Tang, R. Wang, J. Mater. Chem. A 1, 2406 (2013)

[16] Z. Wei, G. Jiang, L. Shen, X. Li, X. Wang, W. Chen, MRS Commun. 3, 145 (2013)

[17] G. Jiang, X. Li, Z. Wei, T. Jiang, X. Du, W. Chen, Powder Technol. 261, 170 (2014)

[18] G. Jiang, X. Li, Z. Wei, T. Jiang, X. Du, W. Chen, Powder Technol. 260, 84 (2014)

[19] G. Jiang, R. Wang, X. Wang, X. Xi, R. Hu, Y. Zhou, S. Wang, T. Wang, W. Chen, ACS Appl. Mater. Interf. 4, 4440 (2012)

[20] V. Balasubramanian, N. Suriyanarayanan, S. Prabahar, Arch. Phys. Res. 3, 88 (2012)

[21] H. Kim, J. Park, K. Kim, M.K. Han, S.J. Kim, W. Lee, Chin. Chem. J. 31, 752 (2013)

[22] Z. Ai, W. Ho, S. Lee, J. Phys. Chem. C 115, 25330 (2011)

[23] B. Topham, M. Kumar, Z. Soos, Chem. Phys. Lett. 493, 251 (2010)

[24] J. Murakam, W. Yamaguchi, Sci. Rep. 2, 407 (2012)

[25] M. Guan, C. Xiao, J. Zhang, S. Fan, R. An, Q. Cheng, J. Xie, M. Zhou, B. Ye, Y. Xie, J. Am. Chem. Soc. 135, 10411 (2013)

[26] N. Farhangi, S. Ayissi, P.A. Charpentier, Nanotechnology 25, 305601 (2014)

[27] M. Khan, W. Cao, J. Li, M.I. Zaman, A. Manan, Int. J. Mod. Phys. B 28, 1450112 (2014)

[28] B. Modak, K. Srinivasu, S.K. Ghosh, J. Phys. Chem. C 118, $10711(2014)$

[29] Y. Inoue, Energy Environ. Sci. 2, 364 (2009)

[30] G. Jiang, B. Tang, X. Li, Z. Wei, X. Wang, W. Chen, J. Wan, L. Shen, Powder Technol. 251, 37 (2014)

[31] S. Vadivel, P. Keerthi, M. Vanitha, A. Muthukrishnaraj, N. Balasubramanian, Mater. Lett. 128, 287 (2014) 